\title{
Localization to atherosclerotic plaque and biodistribution of biochemically derivatized superparamagnetic iron oxide nanoparticles (SPIONs) contrast particles for magnetic resonance imaging (MRI)
}

\author{
Bryan R. Smith • Johannes Heverhagen • \\ Michael Knopp • Petra Schmalbrock • John Shapiro • \\ Masashi Shiomi • Nicanor I. Moldovan • \\ Mauro Ferrari • Stephen C. Lee \\ (C) Springer Science + Business Media, LLC 2007
}

The publisher regrets that the following two figures from the article, "Localization to atherosclerotic plaque and biodistribution of biochemically derivatized superparamagnetic iron oxide nanoparticles (SPIONs) contrast particles for magnetic resonance imaging (MRI)" which appeared in Biomedical Microdevices 9:5, pp. 719-727, should have printed in color. Below are the figures printed in color.

Figures 3 and 5

The online version of the original article can be found at http://dx.doi. org/10.1007/s10544-007-9081-3.

\footnotetext{
B. R. Smith · J. Shapiro • N. I. Moldovan

Department of Biomedical Engineering, Ohio State University,

1080 Carmack Road,

Columbus, OH 43210, USA

J. Heverhagen $\cdot$ M. Knopp $\cdot$ P. Schmalbrock

Department of Radiology, Ohio State University,

630 Means Hall, 1654 Upham Drive,

Columbus, OH 43210, USA

M. Shiomi

Institute for Experimental Animals,

Kobe University Graduate School of Medicine,

Kobe, Japan

M. Ferrari

Brown Institute of Molecular Medicine,

Department of Biomedical Engineering, The University of Texas

Health Science Center,

1825 Pressler, Suite 537D,

Houston, TX 77031, USA

S. C. Lee $(\bowtie)$

Departments of Biomedical Engineering, Chemical and Biomolecular Engineering, Cellular and Molecular Biochemistry. Davis Heart and Lung Research Institute, Ohio State University, 473 W. 12th Ave., Columbus, OH 43210, USA

e-mail: lee.1996@osu.edu
} 

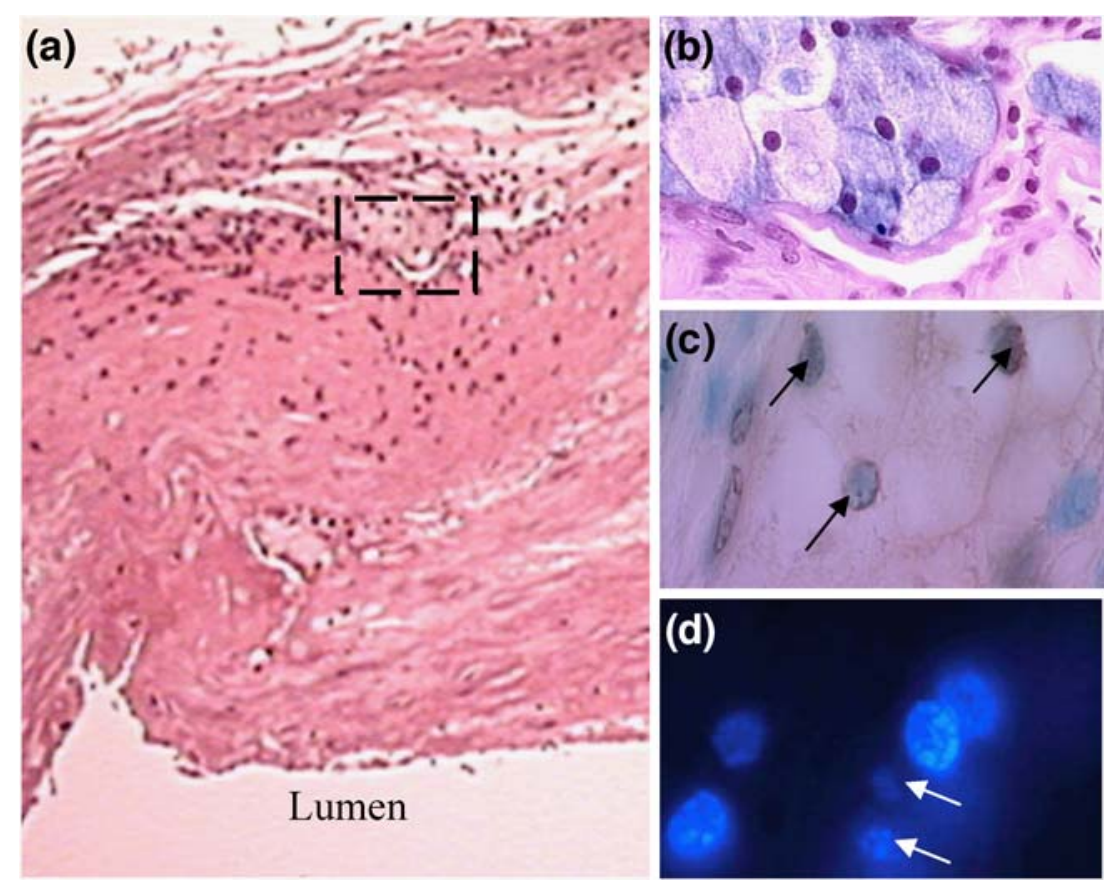

Fig. 3 Annexin V SPIONs are present in the plaque from the imaged regions of abdominal aorta. Histologic section abdominal aorta of the WHHLMI rabbit 1 (see Fig. 2) following imaging is shown. Image is a hematoxylin-eosin stain of a representative section of the region of contrast delivery (40X magnification). An adjacent section was

cells staining with Prussian blue have morphologies characteristic of foamy macrophages. Image c shows TUNEL assay of cells from the same pocket of macrophages (apoptotic nuclei staining brown, indicated with black arrows, non apoptotic nuclei staining with methyl green). Image d shows Hoechst stain of the Prussian-blue staining stained for iron (Prussian blue, image b, 400X magnification). The macrophages with possible fragmented nuclei noted (white arrows)

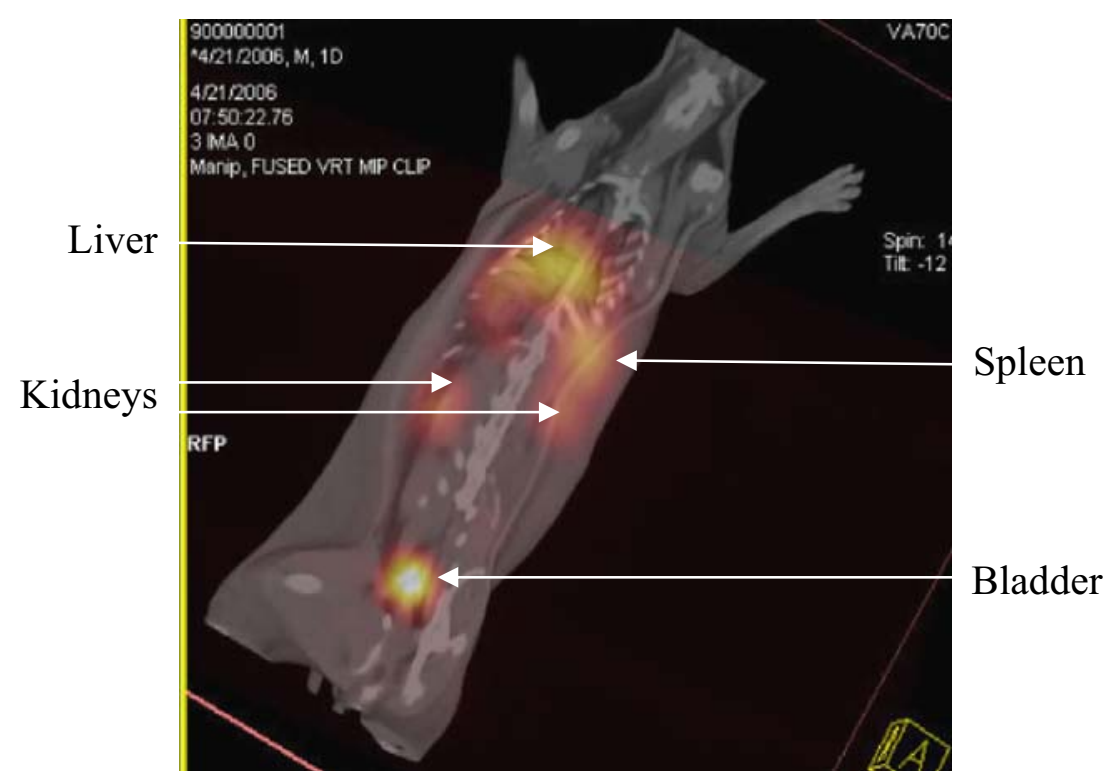

Fig. 5 Most annexin V SPIONs distribute to locations other than the abdominal aorta. Annexin V on SPIONs was labeled with ${ }^{99}$ Tc and injected into a WHHL rabbit. Organs accumulating radiolabel are indicated 